\title{
研究論文
}

\section{External Exposure from the Coning Radioactive Cloud in the Vicinity of Release Point}

\author{
放出点近辺に打ける円錐型放射性雲からの外部被曝
}

\author{
By Toshinori IIJIMA* and Ichiro MIYANAGA*
}

\begin{abstract}
The immersion model based on the meteorological dispersion of the cloud can not be applicable to the estimation of exposure dose near the release point. And it is also important to estimate the exposure of personnel in site of the reactor, and results of the estimation would be useful to know the exposure pattern of ${ }^{41} \mathrm{~A}$ which is released in normal operation of the reactor. In this paper the estimation was made of the external dose hear the release point, comparing with those in immersion case, and the aspect of external dose fom radioactive cloud in whole area of interest was figured out.

The caldulation was carried out under the following assumptions:

(1) The goud is of the coning shape with a vertex angle $20^{\circ}$ which corresponds tof Eategory D (stabiel in Meade and Pasquill's classification.

(2) The kondition of continuity of radioactivity holds and the distribution of the radiogtive concentration is Goussian in the radial direction of the cross section of the cloud.

r.2

The results show that the external dose near the release point from the coning cloud is in the sake order as the maximum dose in immersion case and never exceeds it. Figures were given showing the effect of variation of release point height and iso-dose distribution near the release point. The error resulted from some simplifications of the original equation was within $10 \%$.
\end{abstract}

\section{INTRODUCTION}

In the hazard reports, it is usual to analyse the environmental hazards resulted from the accidental release of fission products under the assumption of MCA (Maximum Credible Accident) of the reactor. Therefore, analysis has been mainly on the hazards of the inhabitants in off-site area which would be at least several hundred meters away from the release point (stack) of radioactive cloud. And the external exposure is estimated with the model of immersion in the cloud covering the people on the ground, diffused according to the meteorological conditions.

This model naturally can not be applied to the analysis of the exposure of the people who would be or work very near to the release point, and if one wants to figure out and gets more detail of the exposure aspect from the radioactive cloud on the whole range of distance from the release point, it is necessary to carry out another calculations though mathematically little more complex. Only one environmental analysis on the NS "Savannah"(1), gives attention about the problem, and takes the same dose as the maximum dose in the immersion case up to the release point without any calculation, by the reason that near the release point the dose should not be less than the maximum dose in the immersion case.

The objectives of this paper are to get the

* 飯島敏哲, 宮水一郎。Radiation Control Office, Health Phys. Div., Japan Atomic Energy Res. Inst. (日本原子力研究所 保健物理部放射楾管理室) 
external dose near the release point, where "immersion model" can not be applied, under some assumptions and see how it would connect with the one in "immersion model". This result may give the level of exposure dose of personnel in site in accidental release of fission products, and also be applicable to the estimation of dose from-say, ${ }^{41} \mathrm{~A}$ cloud which is released in normal operation of a reactor.

\section{Assumptions Taken and Basic Formula of Calculation}

Assumption: (1) The shape of the cloud was taken to be "cone" with a vertex angle $20^{\circ}$ which corresponds to the spreading angle of cloud in the category D of Meade and Pasquill's classification ${ }^{(2)}$, which meteorological condition has $75 \sim 80 \%$ frequency distribution per year in Tokai Area.

Assumption: (2) About the radioactivity in the cone, the condition of continuity holds; that is, the radioactivity in the cross-sectional volume of cloud with unit length be always equal, having the lateral Gaussian distribution of concentration. This would not reflect the exact meteorological diffusion conditions, but for making the calculation simple we took the assumption above.

Now, in Fig. 1, the external dose from the coning cloud to $1 \mathrm{~cm}^{3}$ of air (receiver) at A $\left(x_{0}, y_{0}, 0\right)$ would be expressed as

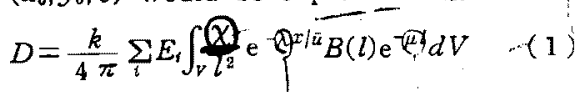

where $k$ : a conversion factor to dose (roentgen) from the concentration $\left(\mathrm{c} / \mathrm{m}^{3}\right)$

$E_{t}: \quad i$-th $\gamma$ ray energy emitted $(\mathrm{MeV})$

Q) the radioactive concentration at $\mathrm{P}$ in the cloud $\left(\mathrm{c} / \mathrm{m}^{3}\right)$

Q. a decay constant

$\bar{u}$ : mean wind velocity $(\mathrm{m} / \mathrm{sec})$

$l$ : distance between elemental volume in cloud and A (m)

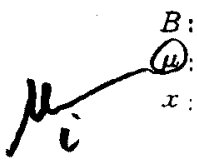

$B$ : build up factor of air absorption coefficient of air distance of the cross-sectional cloud from the release point $(\mathrm{m})$

Radioactive decay is negligible, because $x / \bar{u}$ is so small for $x$ of interest. Build up factor increases and absorption factor $e^{-\mu l}$ decreases as the distance increases, and so these factors were neglected because both factors cancel each other to some extent, and the error coming from this neglection would be less than several percent. (See Appendix)

Then Eq. (1) can be rewritten in the simplfform, in the cylindrical coordinates $x, r$ and $\varphi, x$, being in the wind direction,

$$
D=\frac{k}{4 \pi} \sum_{t} E_{t} \int_{x} \int_{\varphi} \int_{\tau} \frac{\chi}{l^{2}} r d r d \varphi d x
$$

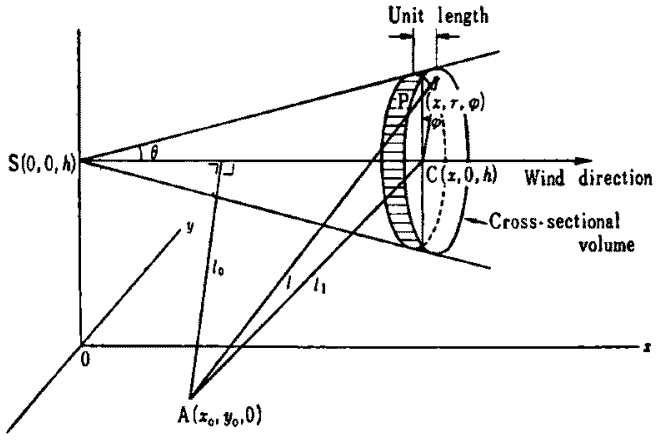

Fig. 1 Schematic figure for the calculation

\section{Calculation of the External Dose}

From assumption (2), the radioactive $x_{i}$ concentration at any point is

$$
\chi=f \exp \left(-a r^{2}\right),
$$

where $r$ is radius of the cross section of the cloud and $f$ is the concentration at the center of cloud. Suppose, at the boundary of the cloud, the concentration be $1 / 10$ that on the central ${ }^{(2)}$.

$$
\chi_{R}=f \exp \left(-a R^{2}\right)=0.1 f, \quad \therefore a=2.30 / R^{2}
$$

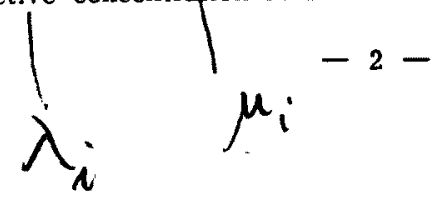


where $R$ is radius of the section and consequently related geometrically to $x$ as

$$
R=x \tan \theta, \quad\left(\theta=10^{\circ}\right) .
$$

In the cross-sectional volume, the below relation holds from assumption(2);

$$
q=\int_{x}^{x+1} \int_{0}^{2 \pi} \int_{0}^{\infty} f \exp \left(-a r^{2}\right) r d r d \varphi d x=\text { const. }
$$

Then we have

$$
q=\pi f / a \text { or } f=a q / \pi \text {. }
$$

In conclusion, the expression of concentration satisfying the assumptions is obtained by substitution of both Eqs. (4) and (7) in (3) as

$$
\chi=\frac{2.30 q}{\pi R^{2}} \exp \left(-2.3 r^{2} / R^{2}\right),
$$

which is function of $r$ and implicitly of $x$, and is independent of the angular variable $\varphi$.

The final equation, substituting the previous results, is

$$
\begin{gathered}
D=\frac{k q}{4 \pi} \sum_{i} E_{i} \int_{0}^{\infty} \int_{0}^{2 \pi} \int_{0}^{\sqrt{2} R} \overline{2}^{2} \cdot 30 \\
\quad \exp \left(-\frac{2 \cdot 30}{R^{2} l^{2}} r^{2}\right) r d r d \phi d x \\
l^{2}=\left(x-x_{0}\right)^{2}+\left(r \sin \varphi-y_{0}\right)^{2}+(h+r \cos \varphi)^{2} .
\end{gathered}
$$

The integration is carried out in tum with respect to each tariable; first the exact integration about $V_{\text {can }}$ be obtained as

$$
\int_{0}^{2 \pi} d \varphi / l^{2}=2 \pi / v^{\prime}\left(l_{1}^{2}+r^{2}\right)^{2}-4 l_{0}^{2} r^{2}
$$

where $\quad l_{1}^{2}=\left(x-x_{0}\right)^{2}+l_{0}^{2} ; l_{0}^{2}=y_{0}^{2}+h^{2}$,

being independent of $r$. As Eq. (9) can not be integrated analytically with respect to $r$, we tried to expand the denominator in Eq. (10) about $\left(r / l_{1}\right)^{2}$; fortunately it can be shown that the error caused from expansion of the equation does not exceed $10 \%$ in maximum when one would take the range of $x_{0}$ not more than $200 \mathrm{~m}$. In integrating with respect to $r$, the upper limit is chosen $\sqrt{2 R}$ instead of infinity, where the concentration is 100th that on the central axis, because, if the upper limit were chosen infinity, the expansion method could not be applied. After integrations, the integrated dose at any receiver due to any total released radioac tive quantity is given by

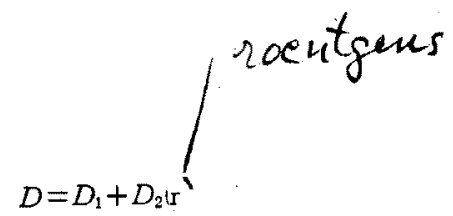

where

$$
\left.\begin{array}{rl}
D_{1}= & 7.89 \times 10^{-2} k q\left(\frac{\pi}{2}+\tan ^{-1} x_{0}\right) l_{0} E_{t} / l_{0} \\
D_{2}= & -1.01 \times 10^{-3} k q \sum E_{i}\left[\frac { l _ { 0 } ^ { 2 } - x _ { 0 } ^ { 2 } } { 4 l _ { 0 } ^ { 2 } } \left\{\frac { 1 } { l _ { 0 } } \left(\frac{\pi}{2}\right.\right.\right. \\
& \left.\left.\left.+\tan ^{-1} \frac{x_{0}}{l_{0}}\right)+\frac{x_{0}}{x_{0}^{2}+l_{0}^{2}}\right\}-\frac{x_{0}^{3}}{2\left(x_{0}^{2}+l_{0}^{2}\right)^{2}}\right]
\end{array}\right\}
$$

In Eq. (11), the first term represents the dose when the all activity were concentrated on the axis, and the second one is considered to be the correction term to this due to the spreading of the cloud. As seen from the equation, the first term is hundred times of the second in order.

The relation between $q$, activity in the sectional volume, and $Q$, total actually released curie, with mean wind velocity $\bar{u}(\mathrm{~m} / \mathrm{sec})$ is

$$
q=Q / \bar{u} .
$$

And if the release rate $Q \mathrm{c} / \mathrm{sec}$ is given in place of the total curie, the dose above will give the exposure dose rate per second.

In order to calculate and compare results with those in immersion case, the following data have been used for estimation.

(1) mean wind speed is $2.5 \mathrm{~m} / \mathrm{sec}$

(2) spreading angle is $20^{\circ}$ in the range of $1 \mathrm{~km}$ (as previously described)

(3) height of stack is taken to be $20 \mathrm{~m}$ and $40 \mathrm{~m}$

(4) total released radioactive quantity is $1 \mathrm{c}$

The absorption coefficient $\mu$, that depends on the $\gamma$ energy, is nearly constant $\left(3.38 \times 10^{-3} / \mathrm{m}\right)$ in the range of $E$ between 0.7 and $1.5 \mathrm{MeV}^{(3)}$, and consequently the conversion factor $k$ is given

$k=$

3. $\left.70 \times 10^{10^{\prime}} \mathrm{dis} / \mathrm{c} \cdot \mathrm{sec}\right) \times 1(\mathrm{MeV} / \mathrm{dis}) \times 3.38 \times 10^{-3}(1 / \mathrm{m})$ $6.77 \times 10^{10}\left(\mathrm{MeV} / \mathrm{m}^{3} \cdot \mathrm{r}\right)$

$=1.85 \times 10^{-3} \mathrm{~m}^{2} \mathrm{r} / \mathrm{c} \cdot \mathrm{sec}$

Variation of the integrated dose at $\mathrm{A}$ along the wind direction is shown in Fig. 2, with parameter $y_{0}$. The upper curve corresponds to height $20 \mathrm{~m}$ and the others are with $43 \mathrm{~m}$. As the change of $y_{0}$ effects in the similar way for the dose as that of $h$, 
this figure can be applied for parameter $h$. $x_{0}$ was taken from negative infinity to $200 \mathrm{~m}$, because the receiver is immersed geometrically in the cloud for $x_{0}$ greater than $240 \mathrm{~m}$, when the stack height is $40 \mathrm{~m}$. When the stack height is $20 \mathrm{~m}, x_{0}$ can not be over $100 \mathrm{~m}$ in

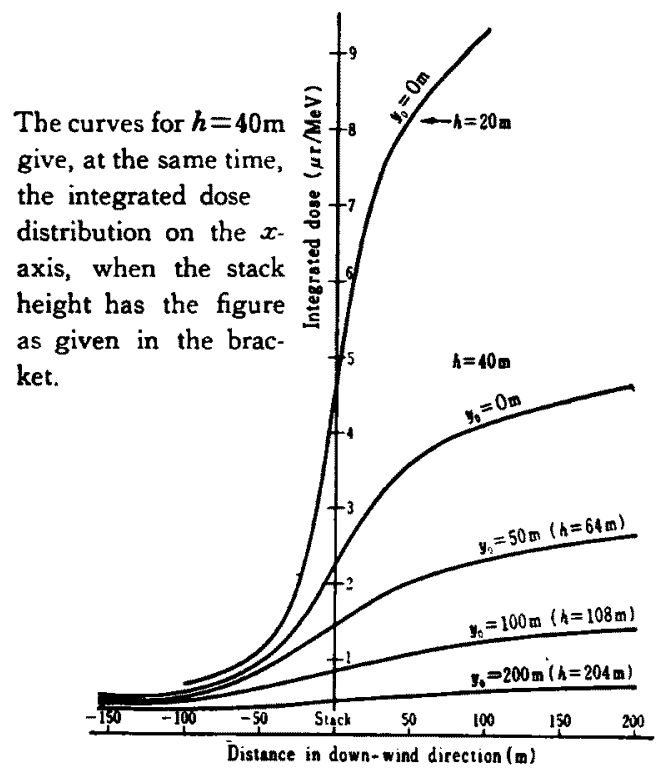

Fig. 2 Distribution of integrated dose in the wind direction

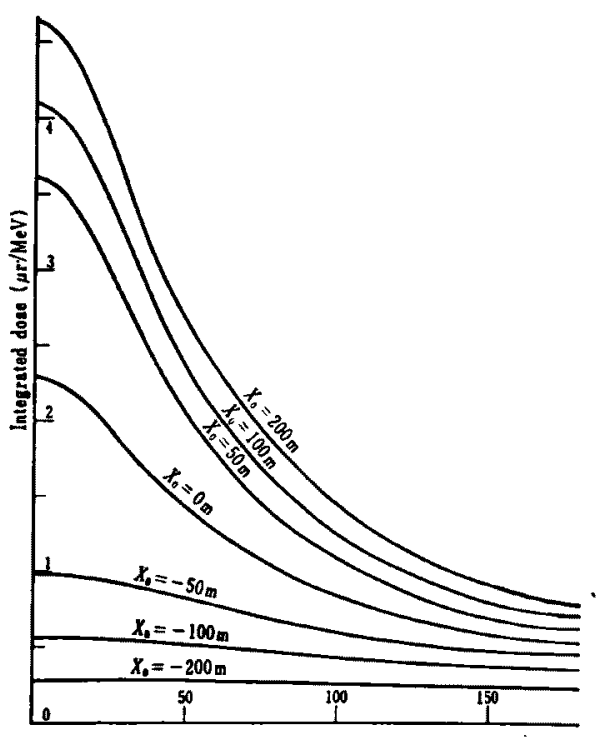

Distance in cross-wind direction ( $m$ )

Fig. 3 Distribution of integrated dose in the cross-wind direction our equation. Any way it can be seen that dose increases rapidly with $h$ decreasing. In Fig. 3 was shown the variation of dose in cross-wind direction. Fig. 4 gives the isodose aspect.

Comparing our results with those obtained in immersion case ${ }^{(\omega)}$, it is found to be conne-

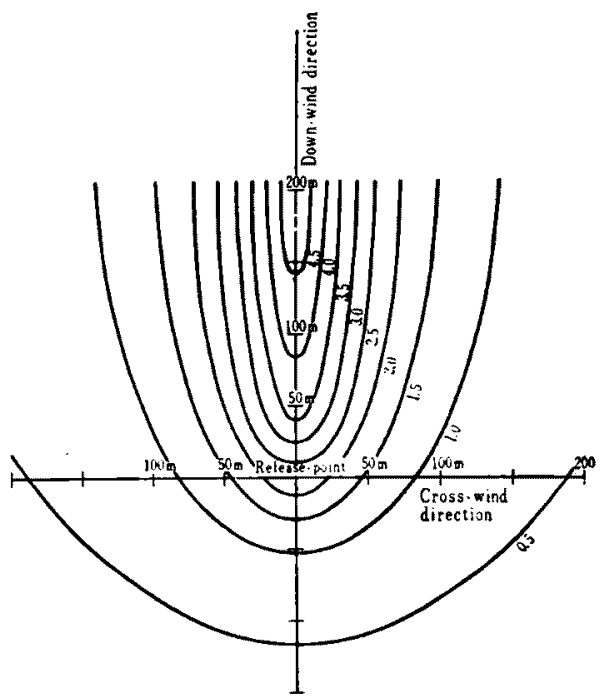

heigh of release-point : $40 \mathrm{~m}$ mean wind speed $: 2.5 \mathrm{~m} / \mathrm{sec}$ stability $: D$ released curie $\quad: 1 \mathrm{c}$ spreading angle $: 20^{\circ}$

Fig. + Iso-dose curve $\left(\mu_{\mathrm{r}} / \mathrm{MeV}\right)$

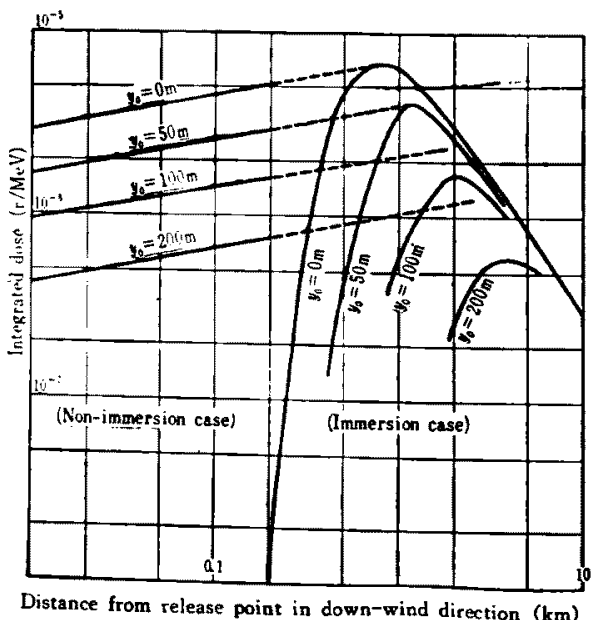

Fig. 5 Exposire dose of non-immersion and immersion case 
.cted in good sense on the $x$-axis but to differ gradually with $y_{0}$ increasing, as shown in Fig. 5.

\section{Conclusion}

(1) The external dose of the coning cloud near the release point was calculated, and compared with the calculation in the immersion case. And Cottrell, et al.'s estimation was verified to be reasonable in practical purpose.

(2) More detail aspects of the external exposure from the radioactive cloud were figured out on the whole distance from the release point.

(3) The result would be applicable also to the estimation of exposure dose of radioargon in the site, which is released in normal operation of the reactor.

We are grateful to Dr. T. Aoki, Director of Health Physics Div. and Mr. S. Sakagishi, Chief of Meteorological and Oceanological Investigation Office, who read the manuscript and gave the useful advices to this study.

(Received July 23. 1962)

\section{REFERENCE}

(1) W B. COTtReLL, et al: ORNL-2867, 21 (1957).

(2) P. J. Meade: Atti del Congresso Scientifico, 315 (1957); Radiological Health Handbook, 145 (1959).

(3) T. AOKI, et al: JAERI-5003, 147 (1961).

(4) J. Z. Holland: Proc. Int. Conf. P. U. A. E., 13, P/572, (1955).

\section{[APPENDIX]}

The errors from neglecting the following factors were estimated in brief.

(1) Decay factor

Since it is impossible to see the behavior of this factor in the integration, only the order of error was estimated. The mean value of $\mathrm{e}^{-\Lambda x}$ is

$$
<\mathrm{e}^{-\Lambda x}>=\frac{1}{L} \int_{0}^{L} \mathrm{e}^{-\Lambda x} d x
$$

where $\Lambda=\lambda / \bar{u}, \lambda$ being a decay constant. Taking ${ }^{11} \mathrm{~A}$, for example; $\lambda=1.05 \times 10^{-1} / \mathrm{m}$, and if $L$, length of the cloud, is taken $500 \mathrm{~m}$,

$$
\left\langle\mathrm{e}^{-\Lambda x}\right\rangle \doteqdot 1
$$

Thus, this may not be considered.

(2) Build up factor

The expression of this factor is given. empirically as ${ }^{(5)}$

$$
B=1+\mu l+(\mu l)^{2} / 7 E^{2.4} .
$$

The third term can be immediately shown to be negligibly small;

$$
\begin{aligned}
& \int \frac{\mu^{2} l^{2}}{7 E^{2.4}} d D=\frac{\mu^{2}}{7 E^{2.4}} \int l^{2} d D<1.62 \times 10^{-8} \\
& \text { for }\left\{\begin{array}{l}
E=1.29 \mathrm{MeV} \\
\mu=3.38 \times 10^{-8} / \mathrm{m} .
\end{array}\right.
\end{aligned}
$$

For the second term, however, the examination is also difficult, and the same way was used as the decay factor. Let $l$ be distance from the central axis of the cloud to the receiver, through rough calculation, then the mean value of $l$ is

$<l>=\int_{0}^{\infty} h \sec \theta d \theta / \int_{0}^{\infty} d \theta ; \tan \alpha=L / h$.

Again taking $L=500 \mathrm{~m}$, then $\alpha=85.5^{\circ}$ for the receiver on the origin and $80.9^{\circ}$ for one on the middle place $(250 \mathrm{~m}$ off from the origin).

$<l>=38 \mathrm{~m}$ for the former and $31 \mathrm{~m}$ for the latter, and consequently

$\langle\mu l\rangle=0.13$ and 0.10 , respectively.

In conclusion, if this factor is considered, the dose increases about $10 \%$.

\section{(3) Absorption factor}

The possible error in the term $\mathrm{e}^{-\mu_{l}}$

will be obtained by substitution of $\langle\mu l\rangle$ above :

$$
\left\langle\mathrm{e}^{-\mu l}\right\rangle=0.88 \text { and } 0.90 \text {, respectively. }
$$

Therefore the decrease of dose due to this factor is about $10 \%$, comparable to that from the build up factor.

After all, the whole error coming from the neglection of the terms above does not exceed $10 \%$, which is mainly contributed from the expansion method. 